\title{
Induced Magnetic Field Effects on Mixed Convection
}

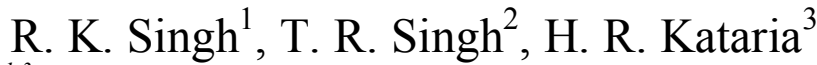 \\ 1,3 Department of Mathematics, M. S. University, Vadodara \\ ${ }^{2}$ Applied Mathematics \& Humanities Department, Sardar Vallabhbhai National Institute Technology, Surat
}

\begin{abstract}
Steady magnetohydrodynamic mixed convective flow of viscous and incompressible fluid in vertical walls having linear axial temperature variation along the surface is studied. Induced magnetic field produced by motion of electric conducting fluid is taken account. The governing partial differential equations are transformed into a system of ordinary differential equations, which is then solved analytically for three different cases depending upon the values of the Rayleigh number and Hartmann Number. Graphical representation of the analytical solution shows that the flow pattern is of parabolic type for low Rayleigh number whereas there is a reverse type flow pattern for higher Rayleigh numbers. The nature of flow pattern can be changed formation changes from parabolic type to reversal type as the value of Rayleigh number increases. The increases strength of magnetic field is to decrease magnitude of fluid velocity, induced magnetic field and skin-friction.
\end{abstract}

Keywords: Magnetohydrodynamics; Mixed convection; Induced magnetic field; Current density.

\section{Introduction}

In recent years, the studies of magnetohydrodynamic flows have attracted many researchers in view of not only its own interest but also due to the applications in astrophysics, geographic and technology. Several electronic equipments designed for airborne applications, satellites, space vehicles and missiles are mostly cooled by forced convection using pressurized air bled off compressor after the hot air is cooled and dehumidified. Long range missiles, cruise missiles, having a flight time of several hours, however, need cooling, which is often done by the liquid fuel they carry. As the magnetic field is known to have an efficient mechanism through which flow and heat transfer in an electrically conducting fluid can be controlled. Hartmann (1937) investigated experimentally as well as theoretically the hydromagnetic flow between two infinite parallel plates. This work provided fundamental knowledge for development of several magnetohydrodynamic (MHD) devices such as MHD pumps, generators, brakes and flow meters.

The first systematic studies of transport processes as a result of the natural and forced convection between two vertical walls, on which temperature very, were obtained by Ostrach [1954, 1955]. Letter on, Beckett [1980], and Beckett and Friend [1984] have discussed the mixed convective flow between vertical walls in the light of numerically and perturbation technique whereas Mishra et al [2002] have studied analytically fully developed mixed convection flow in a porous medium bounded by two vertical walls having a linear axial temperature variation.

Miyatake and Fujii [1873], Nelson and Wood [1989], and Paul et al [1996] have investigated free convection of a viscous and incompressible fluid in a vertical walls under the different thermal conditions on walls.

Jha [1990] have analytically studied the natural convective flow along a vertical infinite plate under a constant magnetic field. Singh and their collaborators have carried out a number of studies on hydromagnetic free convection covering several aspects such as effects of moving boundaries, rotation, asymmetrical heating, porous boundary, etc. [Chandran et al [1993, 1996, 1998], Takhar et al [2002]. In recent years, Singh et al [2010], and Singh \& Singh [2012] have studied effect of induced magnetic field on natural convection in vertical wall and concentric annuli respectively whereas Singh \& Singh [2012] considered magnetohydrdynamic free convective flow past on semi infinite permeable vertical wall.

In the present work, we have analyzed the steady mixed convective flow of a viscous incompressible and electrically conducting fluid between two vertical walls when there is a uniform axial temperature variation along the walls. A constant uniform magnetic field is applied perpendicular to the walls. Depending on the values of the Rayleigh number and magnetic parameter, three different analytical solutions of the governing equations have been obtained. The effects of magnetic field and buoyancy force parameter on the velocity profiles, induced magnetic field, skin- friction and current density have been shown in the graphical/table forms.

\section{Mathematical Formulation Of The Problem}

Consider the steady fully developed flow of a viscous incompressible and electrically conducting fluid in vertical insulated walls under a constant pressure gradient in presence of uniform magnetic field. The walls are separated by a distance $2 L$ and having linear axial temperature variation. We employ a Cartesian coordinate system with origin at the central line of the walls having $x^{\prime}$-axis along the vertical direction and $y^{\prime}$-axis 
perpendicular to the walls. A uniform magnetic field of strength $\left(0, H_{0}, 0\right)$ is applied perpendicular to the walls[Cramer and Pai (1973) and Attia (1998)]. For fully developed laminar flow, the velocity $\left(u^{\prime}\left(y^{\prime}\right), 0,0\right)$ has only a vertical component and is a function of $y^{\prime}$ only whereas magnetic field $\left(H_{x^{\prime}}\left(y^{\prime}\right), H_{0}, 0\right)$ has component $x^{\prime} \& y^{\prime}$ direction respectively. The governing equations of the problem are

$$
\begin{gathered}
-\frac{\partial p}{\partial x^{\prime}}-\rho g+\mu \frac{\partial^{2} u^{\prime}}{\partial y^{\prime 2}}+\mu_{e} H_{0} \frac{\partial H_{x^{\prime}}}{\partial y^{\prime}}=0 \\
H_{0} \frac{\partial u^{\prime}}{\partial y^{\prime}}+\eta \frac{\partial^{2} H_{x^{\prime}}}{\partial y^{\prime 2}}=0 \\
u^{\prime} \frac{\partial T^{\prime}}{\partial x^{\prime}}=\alpha \frac{\partial^{2} T^{\prime}}{\partial y^{\prime 2}}
\end{gathered}
$$

The boundary conditions are

$$
u^{\prime}=H_{x^{\prime}}^{\prime}=0, \quad T^{\prime}=T_{0}^{\prime}+N x^{\prime}, \quad \text { at } \quad y^{\prime}= \pm L
$$

The symbols used in the above expressions have their usual meanings and are defined in the nomenclature.

The Boussinesq approximation is assumed to hold for the evaluation of the gravitational body force and according to which equation of state takes the form

$$
\rho=\rho_{0}\left[1-\beta\left(T^{\prime}-T_{0}^{\prime}\right)\right],
$$

where, $\beta$ is the coefficient of thermal expansion and $\rho_{0}$ and $T_{0}^{\prime}$ are the density and the temperature of the fluid in the reference state.

As a result of uniform axial temperature variation along the walls, the temperature of the fluid can be taken as [Ostrach (1954), Beckett and Friend (1984) and Singh (2002), Singh (2012)]

$$
T^{\prime}-T_{0}^{\prime}=N x^{\prime}+\theta^{\prime}\left(y^{\prime}\right),
$$

where $N$ is a constant temperature gradient.

Using Eqs. (5) and (6), and introducing the non-dimensional quantities (Singh et al [2002])

$$
\begin{aligned}
& y=y^{\prime} / L, \quad u=u^{\prime} / U, \quad U=\alpha P_{x^{\prime}} / L, \quad \theta=\theta^{\prime} /\left(N L P_{x^{\prime}}\right), \quad H=H x^{\prime} / H_{0} \\
& P_{x^{\prime}}=\rho_{0} L^{3}\left[-\left(\frac{1}{\rho_{0}} \frac{\partial P^{\prime}}{\partial x^{\prime}}+g\right)+g \beta N x^{\prime}\right] /(\alpha \vartheta)
\end{aligned}
$$

in Eqs. (1), (2) and (3), we get

$$
\frac{d^{2} u}{d y^{2}}+M^{2} \frac{d H}{d y}+R a \theta=-1
$$

$\frac{d u}{d y}+\frac{d^{2} H}{d y^{2}}=0$

$\frac{d^{2} \theta}{d y^{2}}-u=0$.

In the above equations, additional non-dimensional parameters $M$ and $R a$ denote Hartmann number and Rayleigh number respectively and they are defined as

$$
M=L \mu_{e} H_{0}^{\prime} \sqrt{\sigma / \mu}, \quad R a=N L^{4} g \beta_{0} \rho /(\mu \alpha) .
$$

The non-dimensional boundary conditions are

$$
u=H=\theta=0, \quad \text { at } \quad y= \pm 1 .
$$

Elimination of $H \& \theta$ from Eqs. (8)-(10) has obtained a fourth order differential equation for $u(y)$;

$$
\frac{d^{4} u}{d y^{4}}-M^{2} \frac{d^{2} u}{d y^{2}}+R a u=0 .
$$

The auxiliary roots $m_{1}, m_{2}, m_{3} \& m_{4}$ of the homogenous Eq. (13) are

$$
m_{1}, m_{2}=\sqrt{\left(M^{2} \pm K\right) / 2}, \quad m_{3}, m_{4}=-\sqrt{\left(M^{2} \pm K\right) / 2},
$$


where,

$$
K=\sqrt{M^{4}-4 R a} .
$$

There are arising three different cases for analytical solutions of the differential Eq. (13) and separate solutions are to be obtained for these cases.

Case 1. When $M^{4}>4 R a$

In this case, $M^{2} \pm K$ have positive real values. The roots $m_{1}, m_{2}, m_{3} \& m_{4}$ are real and depend strongly on the values of $M$ and $R a$. The solutions for $u, H \& \theta$ are obtained by solving Eq. (13) with help of Eqs. (9), (10) and (12)

$$
\begin{aligned}
& u=C_{1} \cosh \left(m_{1} y\right)+C_{2} \cosh \left(m_{2} y\right), \\
& H=-\left(\frac{C_{1}}{m_{1}} \sinh \left(m_{1} y\right)+\frac{C_{2}}{m_{2}} \sinh \left(m_{2} y\right)+C_{3} y,\right. \\
& \theta=C_{1}\left[\cosh \left(m_{1} y\right)-\cosh \left(m_{1}\right)\right] / m_{1}^{2}+C_{2}\left[\cosh \left(m_{2} y\right)-\cosh \left(m_{2}\right)\right] / m_{2}^{2}
\end{aligned}
$$

where,

$$
C_{1}=\frac{1}{\left(m_{2}^{2}-m_{1}^{2}\right) \cosh \left(m_{1}\right)}, C_{2}=-\frac{1}{\left(m_{2}^{2}-m_{1}^{2}\right) \cosh \left(m_{2}\right)}, C_{3}=\frac{C_{1}}{m_{1}} \sinh \left(m_{1}\right)+\frac{C_{2}}{m_{2}} \sinh \left(m_{2}\right)
$$

Case 2. When $M^{4}=4 R a$

For this case, auxiliary roots are $\pm M / \sqrt{2}$. The velocity, induced magnetic and temperature fields are derived as

$$
\begin{aligned}
& u=C_{4} \cosh (m y)+C_{5} y \sinh (m y), \\
& H=-\frac{1}{m}\left[C_{4} \sinh (m y)+C_{5}\left(y \cosh (m y)-\frac{\sinh (m y)}{m}\right)\right]+C_{6} y, \\
& \theta=\left\{C_{4}[\cosh (m y)-\cosh (m)]+C_{5}[y \sinh (m y)-\sinh (m)]\right\} / m^{2} \\
& +2 C_{5}[-\cosh (m y)+\cosh (m)] / m^{3},
\end{aligned}
$$

where,

$$
\begin{aligned}
& m=M / \sqrt{2}, C_{4}=[\tanh (m) \sec h(m)] /(2 m), C_{5}=-\sec h(m) /(2 m), \\
& C_{6}=\frac{C_{4}}{m} \sinh (m)+\frac{C_{5}}{m}\left(\cosh (m)-\frac{\sinh (m)}{m}\right)
\end{aligned}
$$

Case 3. When $M^{4}<4 R a$

In this case, As a result of the complex nature of the roots, the expressions for the velocity, induced magnetic and temperature fields are obtained as follows:

$$
\begin{aligned}
& u=2\left[C_{7} \cosh (a y) \cos (b y)+C_{8} \sinh (a y) \sin (b y)\right], \\
& H=\left[C_{9} \sinh (a y) \cos (b y)+C_{10} \cosh (a y) \sin (b y)\right]+C_{11} y, \\
& \theta=I_{1}+I_{2}+I_{3},
\end{aligned}
$$

where, $a=\left[\frac{1}{2} \sqrt{R a}+M^{2} / 2\right]^{1 / 2}, b=\left[\frac{1}{2} \sqrt{R a}-M^{2} / 2\right]^{1 / 2}, a_{0}=a b, a_{1}=a^{2}-b^{2}$,

$C_{7}=1 /\left\{2 a_{0} \cosh (a) \cos (b)[\tanh (a) \tan (b)+\operatorname{coth}(a) \cot (b)]\right\}$,

$C_{8}=1 /\left\{2 a_{0} \sinh (a) \sin (b)[\tanh (a) \tan (b)+\operatorname{coth}(a) \cot (b)]\right\}$,

$C_{9}=\frac{C_{8} b-C_{7} a}{a^{2}+b^{2}}, C_{10}=-\frac{C_{7} b+C_{8} a}{a^{2}+b^{2}}$,

$C_{11}=-C_{9} \sinh (a) \cos (b)-C_{10} \cosh (a) \sin (b)$

$I_{1}=2 C_{7}\left[a_{1} \cosh (a y) \cos (b y)+a_{0} \sinh (a y) \sin (b y)\right] /\left(a^{2}+b^{2}\right)^{2}$, 


$$
\begin{aligned}
& I_{2}=2 C_{8}\left[a_{1} \cosh (a y) \sin (b y)-a_{0} \sinh (a y) \cos (b y)\right] /\left(a^{2}+b^{2}\right)^{2}, \\
& I_{3}=-2\left\{a_{1} \cosh (a)\left[C_{7} \cos (b)+C_{8} \sin (b)\right]\right. \\
& \left.+a_{0} \sinh (a)\left[C_{7} \sin (b)-C_{8} \cos (b)\right]\right\} /\left(a^{2}+b^{2}\right) .
\end{aligned}
$$

Due to symmetry in flow, skin-friction in non-dimensional from for different cases, on the both walls, will be same and have been obtained by following relation

$$
\tau=\left(\frac{d u}{d y}\right)_{y=1} .
$$

By doing so the expressions for the skin friction for different cases have been obtained as follows:

Case 1. Skin-friction when $M^{4}>4 R a$

$$
\tau=C_{1} m_{1} \sinh \left(m_{1}\right)+C_{2} m_{2} \sinh \left(m_{2}\right)
$$

Case 2. Skin-friction when when $M^{4}=4 R a$

$$
\tau=\left(C_{4} m+C_{5}\right) \sinh (m)+C_{5} m \cosh (m)
$$

Case 3. Skin-friction when when $M^{4}<4 R a$

$$
\tau=2\left[\left(a C_{7}+b C_{8}\right) \sinh (a) \cos (b)+\left(a C_{8}-b C_{7}\right) \cosh (a) \sin (b)\right]
$$

Current density $\left(0,0, J_{z}\right)$

$$
J_{z}=\frac{-1}{4 \pi} \frac{\partial H}{\partial y}
$$

for different cases have been obtained as:

Case 1. Current Density when $M^{4}>4 R a$

$$
J_{z}=\frac{1}{4 \pi}\left(C_{1} \cosh \left(m_{1} y\right)+C_{2} \cosh \left(m_{2} y\right)-C_{3}\right)
$$

Case 2. Current density when $M^{4}=4 R a$

$$
J_{z}=\frac{1}{4 \pi}\left(C_{4} \cosh (m y)+C_{5} y \sinh (m y)-C_{6}\right)
$$

Case 3. Current density when $M^{4}<4 R a$

$$
J_{z}=-\frac{1}{4 \pi}\left[\left(a C_{9}+b C_{10}\right) \cosh (a y) \cos (b y)+\left(-b C_{9}+a C_{10}\right) \sinh (a y) \sin (b y)+C_{11}\right] .
$$

\section{Results And Discussion}

The non-dimensional parameters in the model are $R a$, the Rayleigh number and $M$, the Hartmann number. The outcome from the analytical solution is given in graphical form regarding to the cases $M^{4}>=<4 R a$. The velocity profiles, for small values of Rayleigh number, are shown in figures 1-3 corresponding to the above mentioned cases respectively. It is clear from the figures that symmetric flow about $y=0$ occurs between the walls, for all considered values of $R a \& M$. The nature of velocity profiles for third case is different from first two cases. Here, in $3^{\text {rd }}$ case, maximum velocity of fluid is shifted near to walls (Fig. 3 ; curves $2,4 \& 6$ ). These figures show that the flow is parabolic type up to certain values of the low Rayleigh number and thus the flow formation is due to the forced convection. The nature of flow formation changes from parabolic type to reversal type as the value of Rayleigh number increases (Fig. 2: curves $3 \&$ 4; Fig. 3: curves 2, $4 \& 6$ ). Further, we observed that the effect of the increasing strength magnetic field is to decrease the velocity profiles for all considered cases.

The variations in velocity profiles, for higher values of Rayleigh, have been depicted in Figs 4-6 respectively for all cases. The velocity profiles are higher near the walls compared to middle region. Fig. 4 shows that differences between magnitude velocity in the middle region and near the walls is higher for large values of $R a$ compare to small values of $R a$. Fig. 6 shows (Curves 2, 4, $5 \& 6$ ) that the velocity profiles are maximum near the walls and thereafter having decreasing tendency and they have values very closed to zero near middle region. The effect of increasing strength of magnetic field, for $R a=500$ (Curves $1,3 \& 5$ ), is to decelerate fluid velocity near the walls and accelerate near the middle region. Furthermore, from Fig. 6 , for large 
value of Rayleigh number, the velocity profiles of fluid do not alter with the magnetic strength (As curves 4,5 $\& 6$ coincide). It is clear from Figs. 4, 5 and 6 that the point of maximum velocity profiles tends to sift nearer to the walls as values of $R a$ is increased. In all the cases, near the walls, the velocity of fluid is maximum and thus the flow is dominated by the free convection. Thus, the forced convection dominated flow can be changed into the free convection dominated flow by increasing the values of Rayleigh number.

Figs. 7-9 show the induced magnetic field profiles for various low values of Rayleigh number and Hartmann Number. We observed that induced magnetic field is upward direction in first half portion and downward direction in second half portion between the walls. Similar trend of the induced magnetic field observed by Singh et al [2010] and Singh [2012]. Since the velocity of fluid decrease with $R a \& M$, therefore magnitude of induced magnetic field also decrease as $R a \& M$ increase.

Figures 10-12 display the induced magnetic field profiles which developed in vertical walls for higher Rayleigh numbers. It is observed that magnitude of induced magnetic field maximum near at $y=-0.5 \& 0.5$. The magnitude of induced magnetic field decrease as increases Rayleigh number and strength of magnetic field. The region of this behavior is that the effect of velocity on $\mathrm{H}$ is indirect.

The effects of the Rayleigh number and Hartmann number on skin-friction $\tau$ at the wall $y=1$ are shown in Fig 13. We observed that skin-friction decreases as increases as the strength of the magnetic field and Rayleigh number increases.

\section{Conclusion}

The present study investigates the fully developed mixed convective flow of an incompressible viscous electrically conducting fluid between vertical walls for case variable surface temperature in presence of uniform magnetic field. Solutions for the velocity, induced magnetic field temperature and skin friction have been derived by solving a fourth order differential equation for three different cases depending on the values of the physical parameters of the model. Flow formation changes form forced convection dominates to free convection dominates as Rayleigh's number increases. Drag-like force increases as the strength of the magnetic field (represented by Hartmann number M) increases producing reductions in the fluid velocity. Since velocity decreases as magnetic strength increases, so skin friction and induced magnetic field have also decreasing tendency with increasing magnetic field.

\section{Nomenclature}

$H_{0}^{\prime} \quad$-constant magnetic field flux density

$g$-acceleration due to gravity

$J \quad$-current density

$K$-defined as equation (14)

$M$-Hartmann number

$N$-temperature gradient constant

$P_{x^{\prime}} \quad$-dimensionless quntity

$R a \quad$-Rayleigh number

$T^{\prime}$-temperature

$T_{0}^{\prime}$-temperature in reference state

$u^{\prime}$-velocity of fluid along the $x^{\prime}$ axis

$u$-fluid velocity in non-dimensional form

$x^{\prime}, y^{\prime} \quad$-Cartesian coordinates

$x, y \quad$-dimensionlees Cartesian coordinate

\section{Greek symbols}

$\alpha$-thermal diffusivity

$\beta$-coefficient of thermal expansion

$\theta^{\prime} \quad$-temperatuer

$\theta$-dimensionless temperature

$\mu$-dynamic viscosity of the fluid

$\vartheta \quad$-kinematic viscosity of the fluid 
$\rho$-fluid density

$\sigma$-electrical conductivity

$\tau \quad$-Skin-friction in non-dimensional form at wall $y=1$

\section{References}

[1]. Hartmann, J.: Hg-dynamics I theory of the laminar flow of an electrically conductive liquid in a homogenous magnetic field, Det Kal. Danske Videnskabernes selskab, Mathematisk-fysiske Meddeleser. XV, 1-27 (1937).

[2]. Ostrach, S.: Combined Natural and Forced Convection Laminar Flow and Heat Transfer of Fluids With and Without Heat Sources in Channels with Linearly Varying Wall Temperature, NACA Technical Note No. 3141(1954).

[3]. Ostrach, S.: Unstable convection in vertical channels with heating form below including effects of heat sources and frictional heating, NACA Technical Note No. 3458(1955).

[4]. Beckett, P. M.: Combined natural and forced convection between parallel walls, SIAM, Journal Applied Mathematics, 39(2), 372-384(1980).

[5]. Beckett, P. M., Friend, I. E.: Combined natural and forced convection between parallel walls: developing flow at higher Rayleigh numbers, International Journal of heat and Mass Transfer, 27, 611-621(1984).

[6]. Singh, A. K., Mishra, A. K., Paul, T.: Mixed convection flow in a porous medium bounded by two vertical walls, Forschung im Ingenieurwesen, 67(5), 198-205(2002).

[7]. Aung, W., Fletcher, L. S., Sernas, V.: Developing laminar free convection between vertical flat plates with asymmetrjc heating, International Journal of Heat Mass Transfer 15, 2293-2308(1972).

[8]. Miyatake, O., Fujii, T.: Natural convection heat transfer between vertical parallel plates at unequal uniform temperatures, Heat Transfer-Japanese. Research, 2, 79-88(1973).

[9]. Nelson, D. J., Wood, B. D.: Combined heat and mass transfer natural convection between vertical parallel plates, International Journal of Heat Mass Transfer, 32(9), 1779-1787(1989).

[10]. Paul, T., Jha, B. K., Singh, A. K.: Transient free convective flow in a vertical channel with constant temperature and constant heat flux on walls, Heat and Mass Transfer, 32, 61-63(1996).

[11]. Jha, B. K.: MHD free-convection and mass transform through a porous media, Astrophysics and Space Science, 175, 283289(1990).

[12]. Chandran, P., Sacheti, N. C., Singh, A. K.: Effects of rotation on unsteady hydrodynamic Couette flow, Astrophysics and Space Science, 202, 1-10(1993).

[13]. Chandran, P., Sacheti, N. C., Singh, A. K.: Haydromagnetic flow and heat transfer past a continuously moving porous boundary, International Communications in Heat and Mass Transfer, 23, 889-898(1996).

[14]. Chandran, P., Sacheti, N. C., Singh, A. K.: Unsteady hydromagnetic free convection flow with heat flux and accelerated boundary motion, Journal of Physical Society of Japan, 67, 124-129(1998).

[15]. Singh, A. K., Chandran, P., Sacheti, N. C.: Effects of transverse magnetic field on a flat plate thermometer problem, International Journal of Heat and Mass Transfer, 43, 3253-3258(2000).

[16]. Chandran, P., Sacheti, N. C., Singh, A. K.: An undefined approach to analytical solution of a hydromagnetic free convection flow, Scientiae Mathematicae Japonicae, 53, 467-476(2001).

[17]. Singh, R. K., Singh, A. K., Chandran, P., Sacheti, N. C.: Effect of Induced magnetic field on hydromagnetic free convective flow between vertical walls, Heat and Mass transfer, 46, 523-529(2010).

[18]. Singh, R. K., Singh, A. K.: Effect of induced magnetic field on natural convection in vertical concentric annuli, Acta Mechanica Sinica, 28(2), 315-323(2012).

[19]. Singh, R. K., Singh A. K.: MHD free convective flow past a semi-infinite vertical permeable wall, Applied Mathematics and Mechanics, 33(9), 1207-1222(2012).

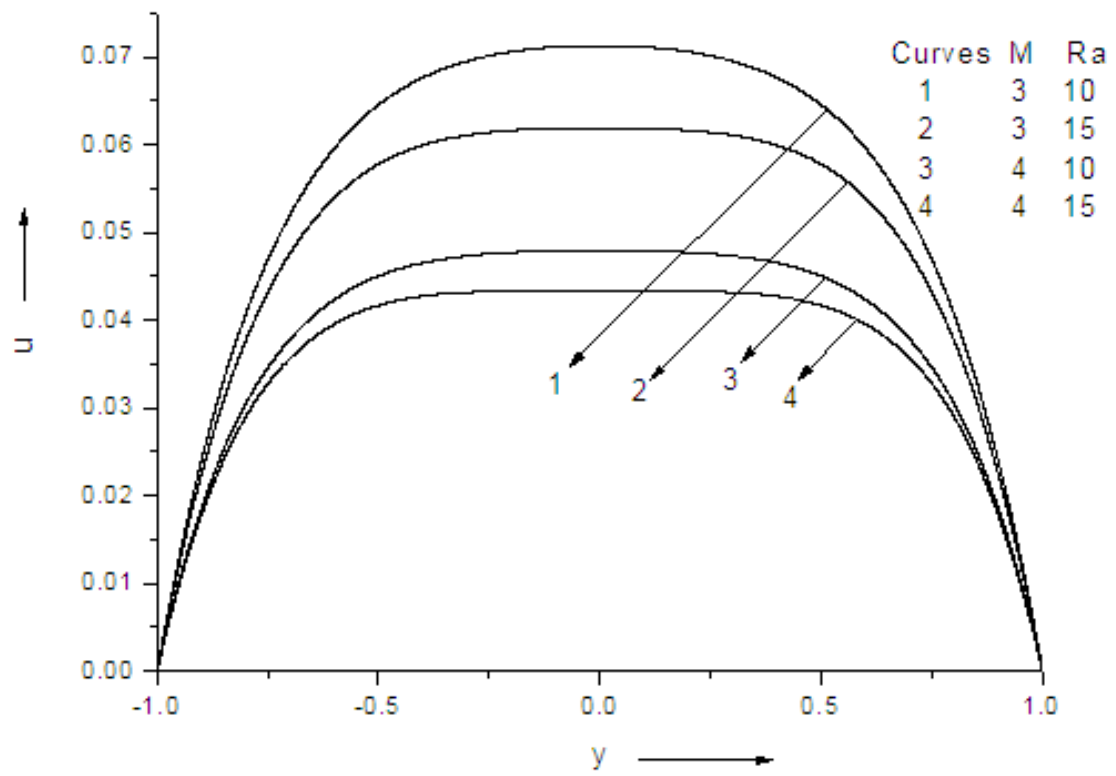

Fig.1. Velocity profile for $M^{4}>4 R a$. 


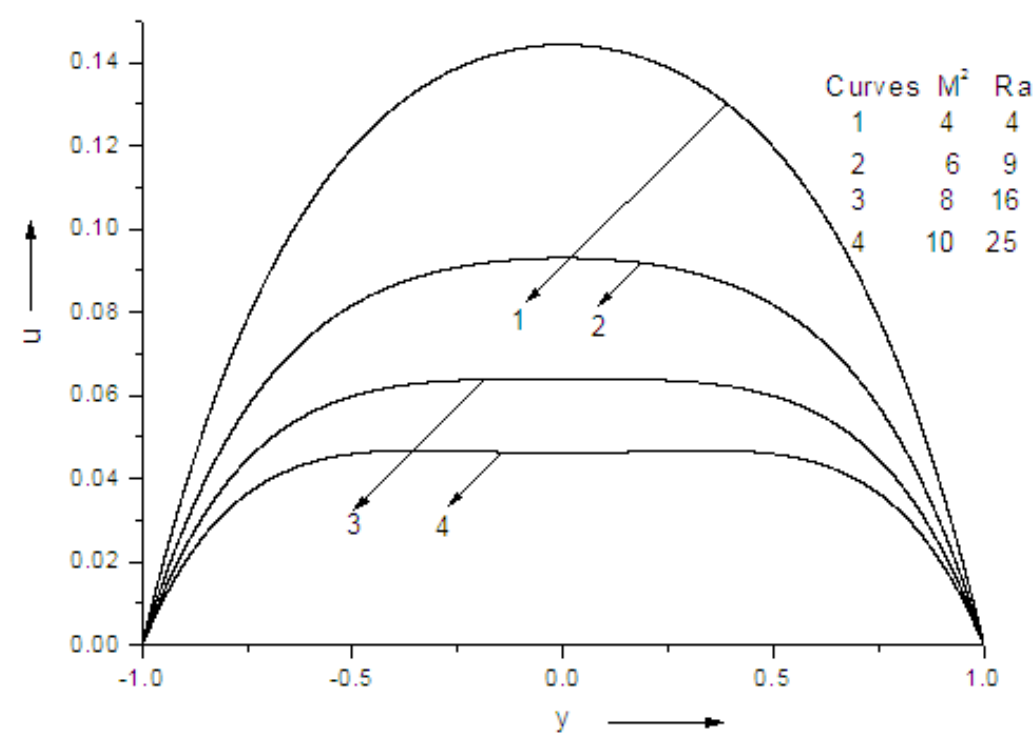

Fig. 2. Velocity profile for $M^{4}=4 R a$.

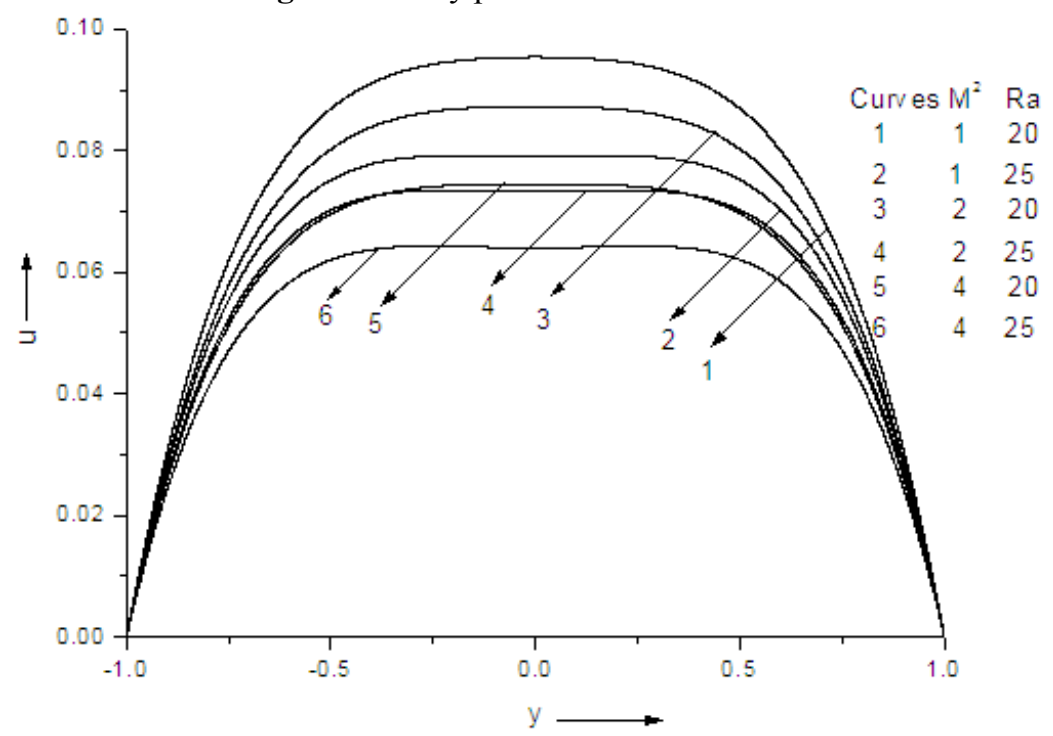

Fig. 3. Velocity profile for $M^{4}<4 R a$.

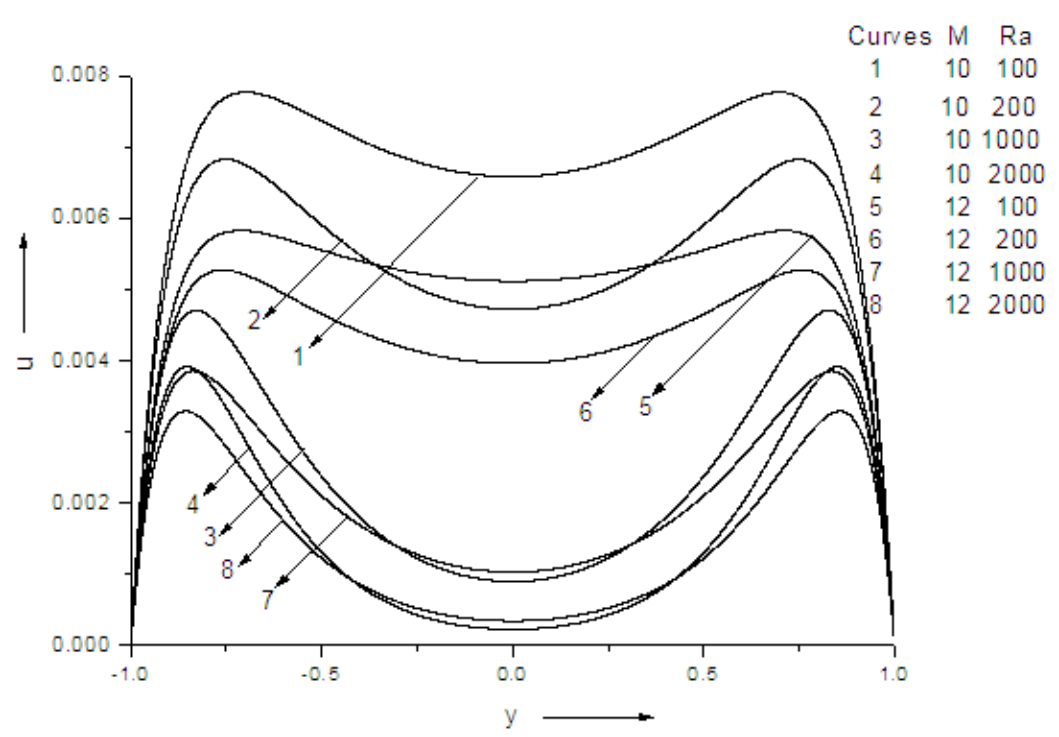

Fig.4. Velocity profile for $M^{4}>4 R a$. 


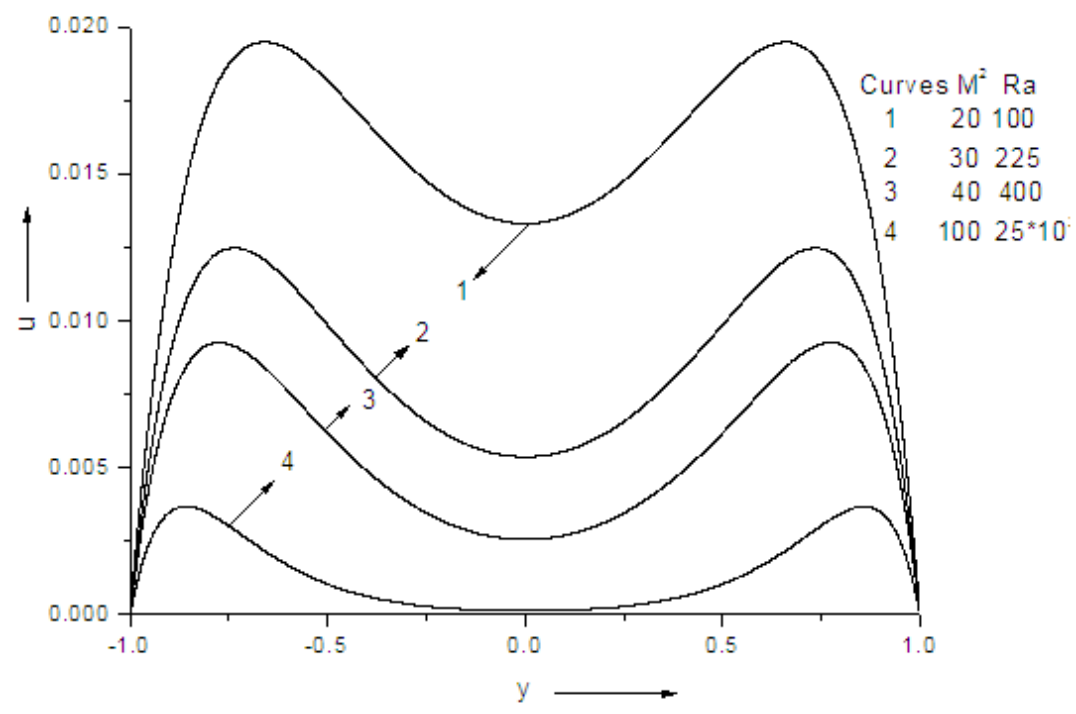

Fig.5. Velocity profile for $M^{4}=4 R a$.

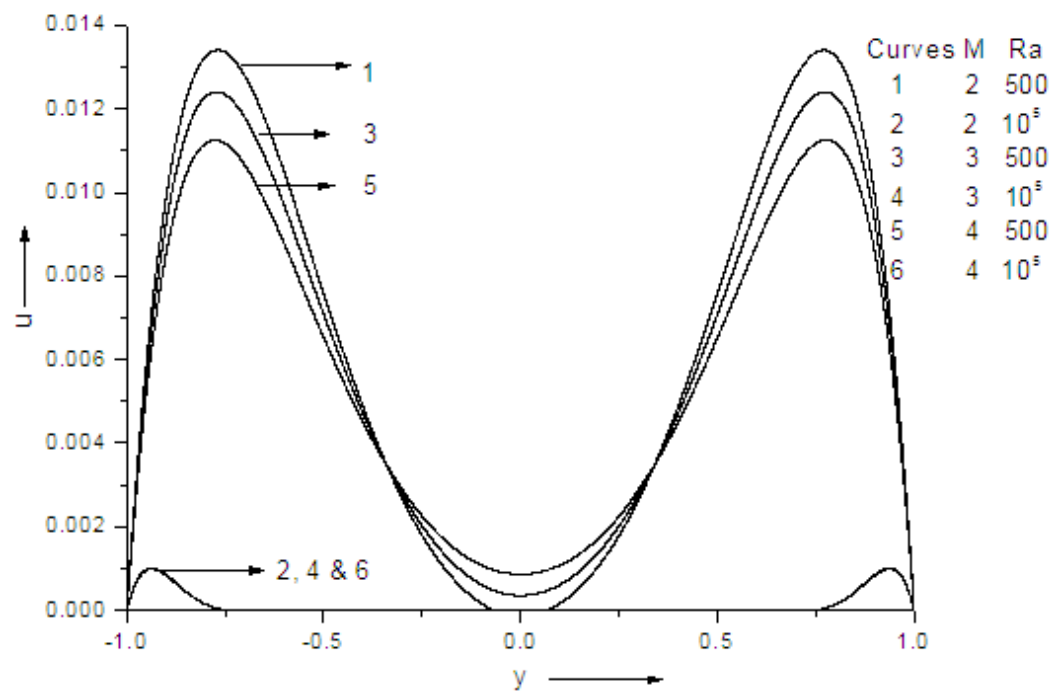

Fig.6. Velocity profile for $M^{4}<4 R a$.

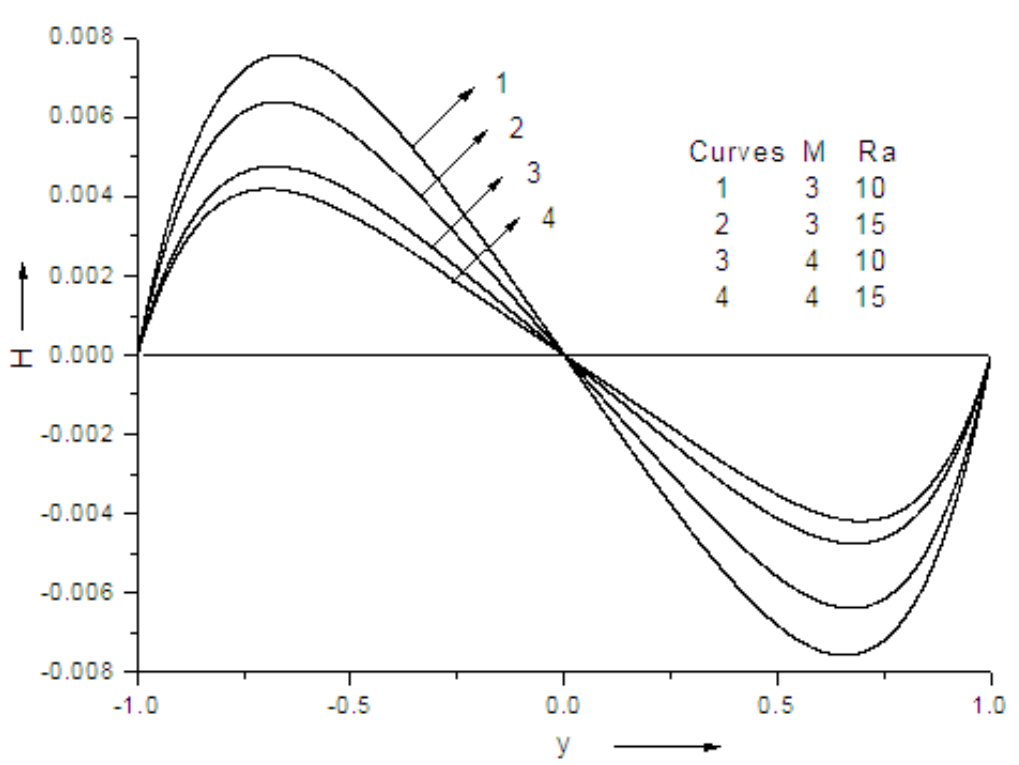

Fig.7. Induced magnetic field for $M^{4}>4 R a$. 


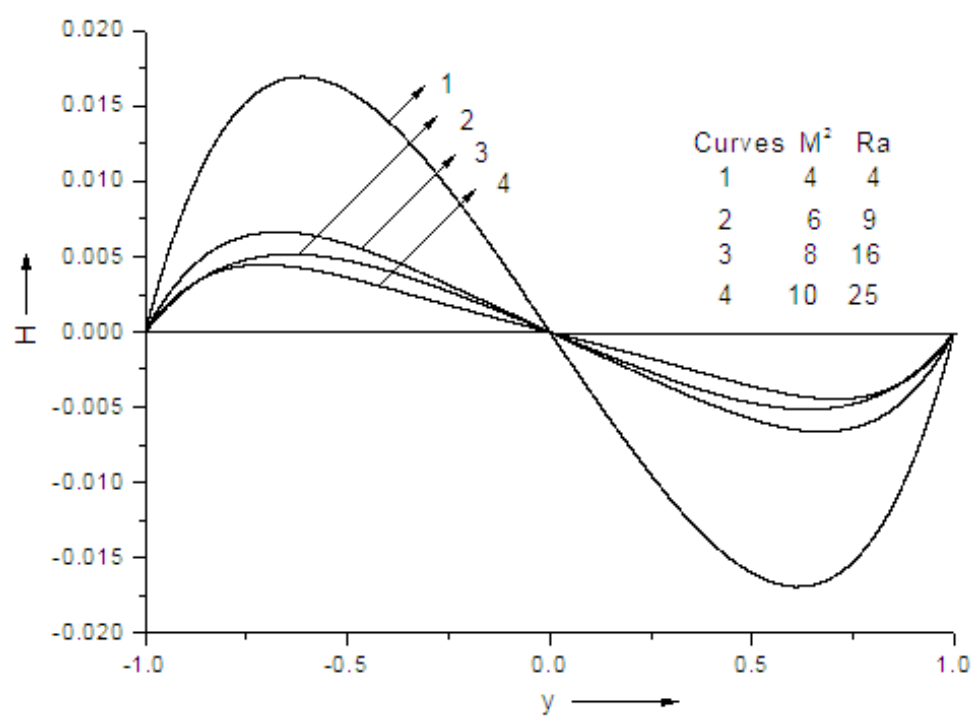

Fig.8. Induced magnetic field for $M^{4}=4 R a$.

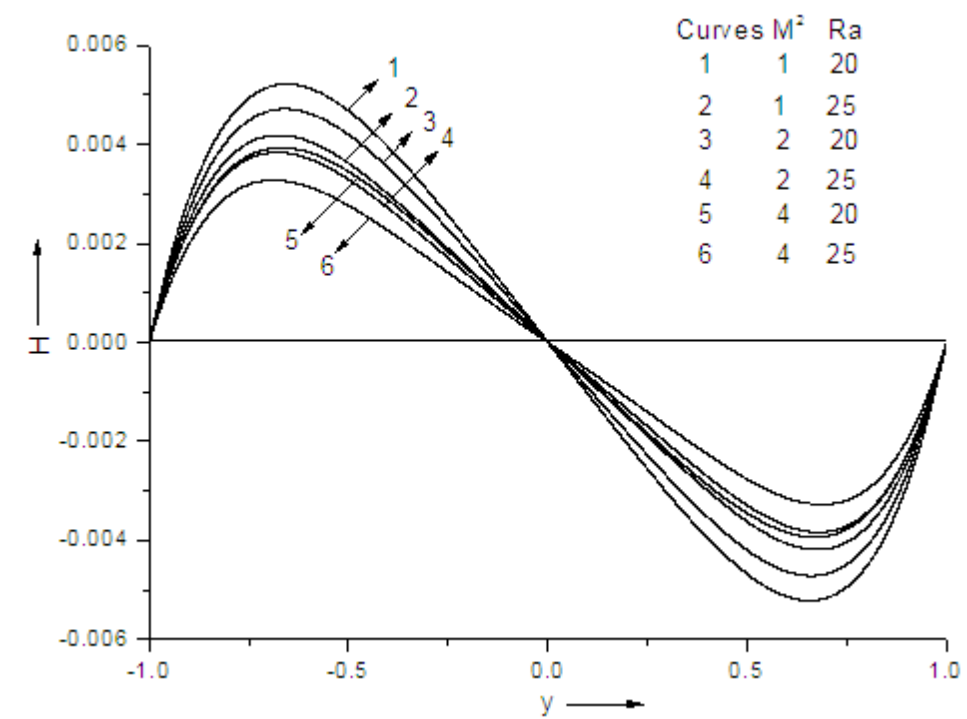

Fig.9. Induced magnetic field for $M^{4}<4 R a$.

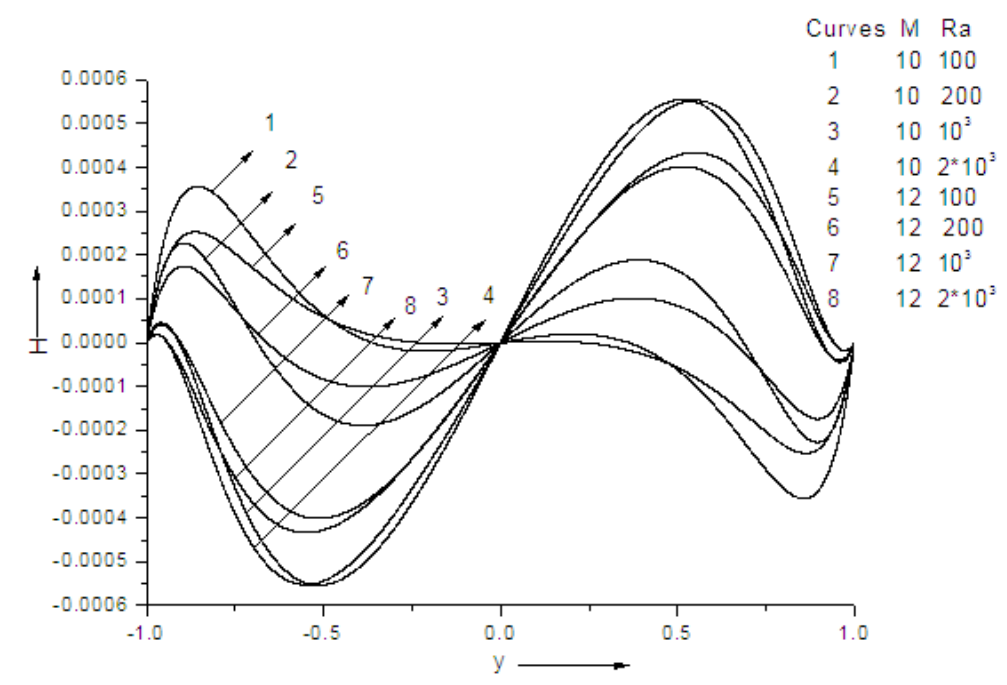

Fig.10. Induced magnetic field for $M^{4}>4 R a$. 


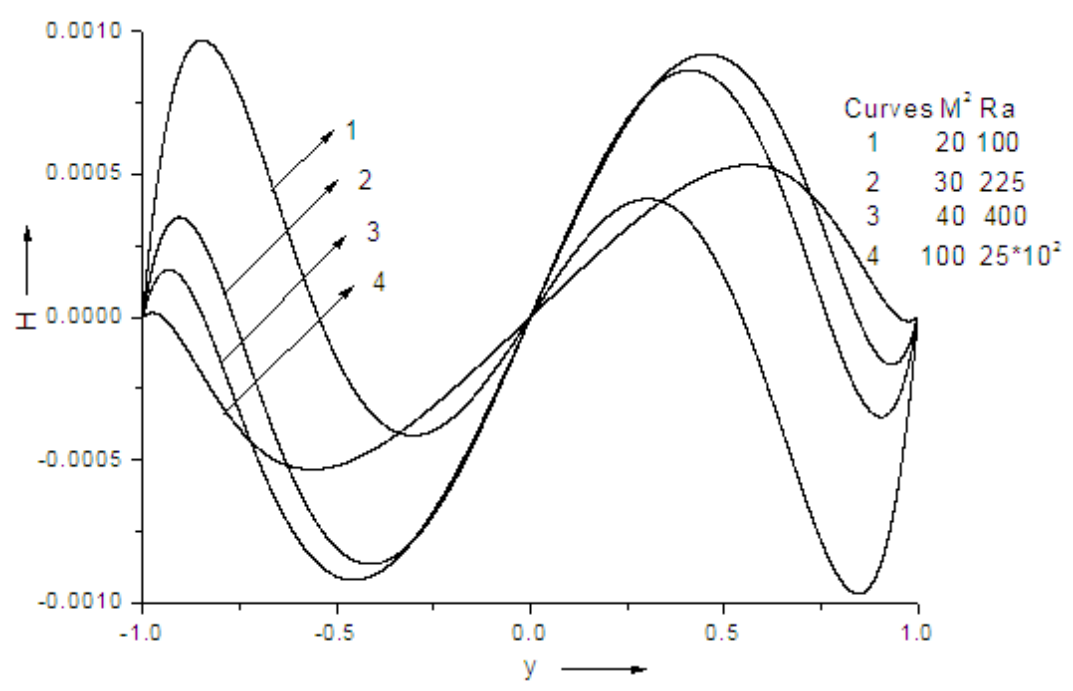

Fig.11. Induced magnetic field for $M^{4}=4 R a$.

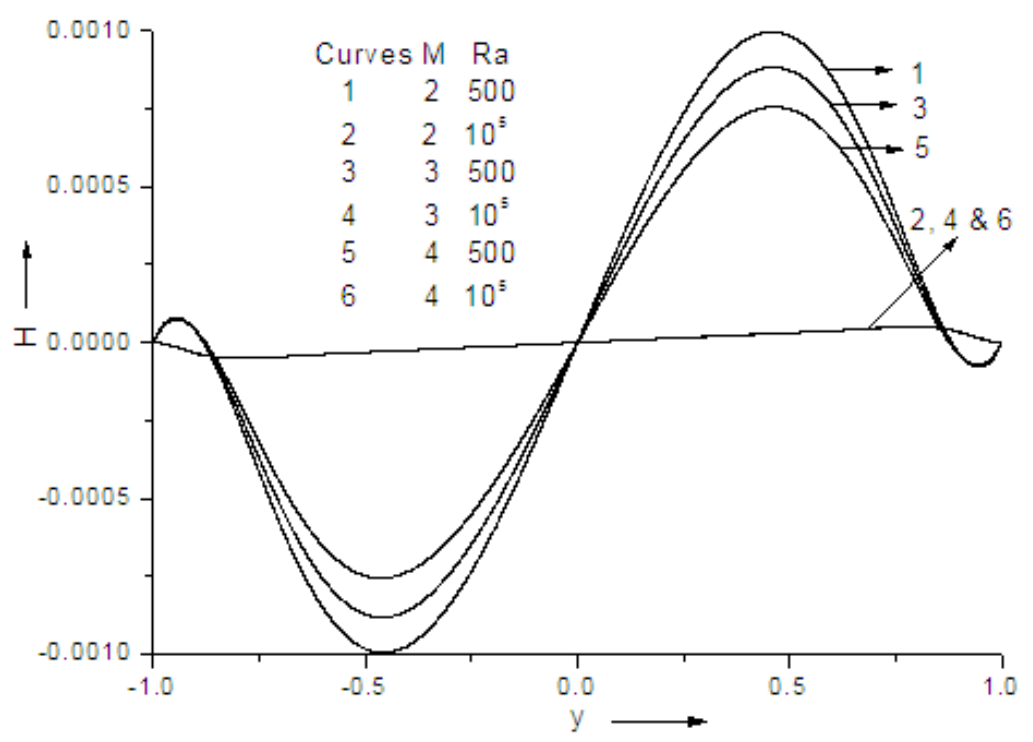

Fig.12. Induced magnetic field for $M^{4}<4 R a$.

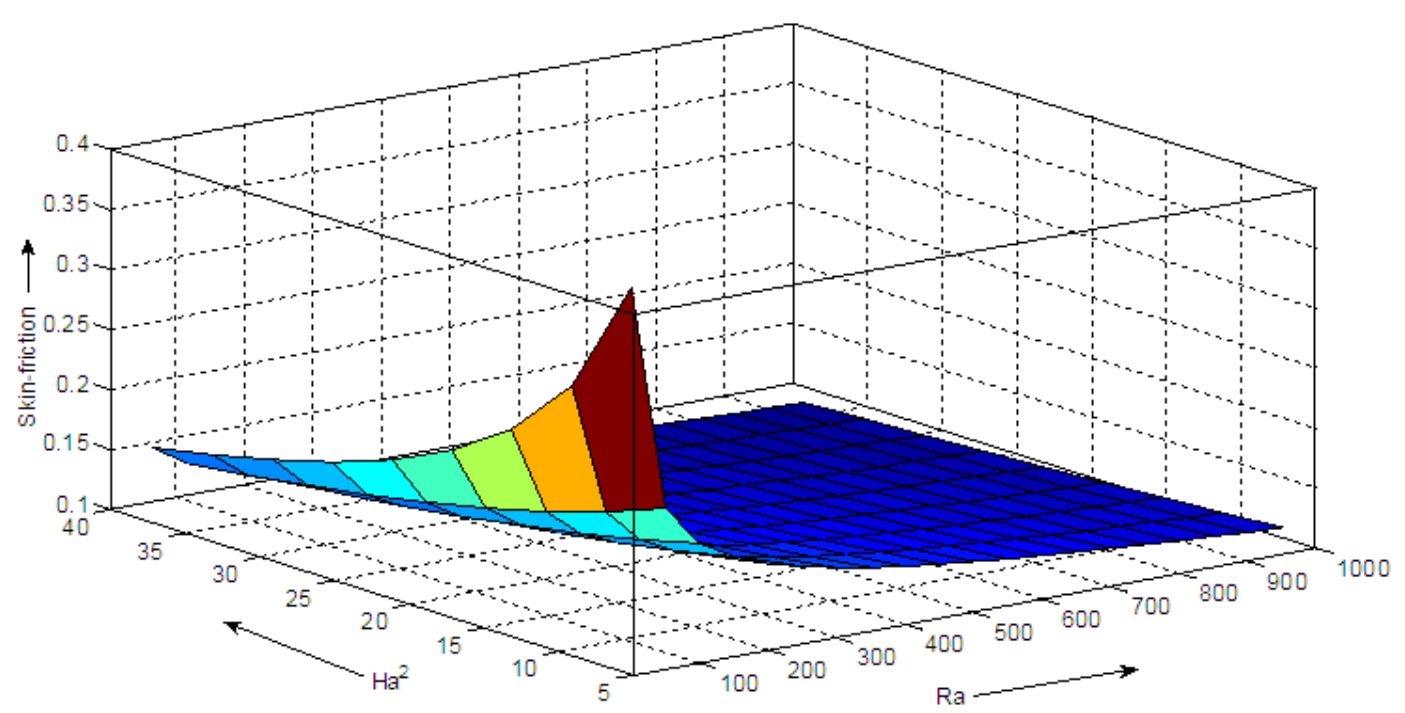

Fig. 13. Skin-friction for $M^{4}>=<4 R a$ 